\title{
Editorial
}

\section{Complex Systems Modelling, Analysis, and Control}

\author{
Constantinos I. Siettos, ${ }^{1}$ Yannick De Decker, ${ }^{2}$ and Georgios C. Georgiou ${ }^{3}$ \\ ${ }^{1}$ School of Applied Mathematics and Physical Sciences, National Technical University of Athens, 15780 Athens, Greece \\ ${ }^{2}$ Center for Nonlinear Phenomena and Complex Systems (CENOLI), Université Libre de Bruxelles, Campus de la Plaine CP231, \\ Brussels, Belgium \\ ${ }^{3}$ Department of Mathematics and Statistics, University of Cyprus, P.O. Box 20537, 1678 Nicosia, Cyprus
}

Correspondence should be addressed to Constantinos I. Siettos; ksiet@mail.ntua.gr

Received 24 October 2013; Accepted 24 October 2013

Copyright (c) 2013 Constantinos I. Siettos et al. This is an open access article distributed under the Creative Commons Attribution License, which permits unrestricted use, distribution, and reproduction in any medium, provided the original work is properly cited.

Complexity pertains to the structure of many real-world systems, shaping their behaviour and dynamics. Over the last few years, there has been an increasing interest in studying the emergent complex dynamics of processes ranging from fluid mechanics and materials science to the internet and social networks and from biological and neural systems to epidemic and ecological systems. Due to the strongly heterogeneous character of such systems as well as the stochastic and nonlinear very large scale interactions over complex networks, the emergent (macroscopic) behavior is most of the times far from trivial to predict. Self-organization, sustained oscillations, travelling waves, multiplicity of stationary states, and spatiotemporal chaos are paradigms of the rich nonlinear behavior at the coarse-grained systems level.

Hence, the quest for developing new modelling, computational-assisted, and control methodologies to analyze and design the behaviour of complex systems appears to be a major and timely challenge of our times. Finding and developing such methodologies would enable a better understanding, prediction, and design of complex systems with important health, social, and economic impact. In this special issue, we invited authors to contribute with original research and review articles with a strong focus on these topics including materials science, fluid mechanics, stochastic modelling of complex problems, multiscale computational methods, topology of complex networks and their dynamics, control and optimization methods for multiscale/complex systems, bifurcation analysis of microscopic and large scale systems, computational epidemiology, and computational neuroscience.

We received a total of 59 paper. All papers underwent a very careful peer review process and were revised to accommodate the comments made by the reviewers. We gave a lot of attention to the selection of the appropriate reviewers. We based our choice on strict academic criteria including the expertise in the field of each submitted paper and international reputation. In a very small number of manuscripts for which we received conflicting pieces of advice we chose to submit the corresponding manuscript to other referees and finally we made our decision taking into account all the reports and the strength of the arguments therein on the basis of academic merit and subject appropriateness. Finally, 14 manuscripts were accepted, that is, the acceptance rate was slightly less than $24 \%$. The special issue is freely available online to all interested readers.

In what follows we briefly describe the published contributions.

M. Liu et al. develop a deadlock prevention system with resources reallocation and supervisor reconfiguration for designing near-optimal supervising controllers for automated flexible manufacturing systems. Their methodology is mainly focused on manufacturing-oriented Petri nets.

L. Chai and S. Fei address the problem of stabilization of a class of linear time-delayed systems for which the delay parameter appears in both the control and system matrices and is unknown. The authors show how one can 
design a feedback controller based on the "error to error" and separated "descriptor form" functional technique to stabilize the underlying systems and provide the relevant sufficient conditions which can be obtained by a solvable linear matrix inequality problem.

Xian et al. introduce a control system that can be used to suppress epileptiform spikes from a regular network of coupled neural populations. For their illustrations they used a Jansen's neural mass model. They show through simulations that the type of the controlled populations, the number of the controlled populations and the control gain are important factors that affect the performance of the control system.

C.-Y. Huang et al. describe a (four-layer universal epidemic dynamics) FLUed platform incorporating complex daily commuting network data into multiple age-structured compartmental models for the simulation and the analysis of the dynamics of epidemics. The proposed model was validated and tested through real-world data concerning the seasonal influenza A and 2009 swine-origin influenza A (H1N1). The potential impact of intervention policies as well as variation in initial outbreak areas for novel/seasonal influenza A in Taiwan is also investigated and discussed.

Xiangjie Liu et al. design a fuzzy state feedback control system based on the delay partitioning approach in order to examine the passivity of Tagaki-Sugeno fuzzy systems with time delay. The resulting sufficient conditions are given in the form of linear matrix inequalities.

In the paper of P. Liu et al., a proportional-integralderivative (PID) controller is designed with a feedforward compensation for the trajectory tracking problem of a giant magnetostrictive actuator (GMA). The effectiveness of their proposed approach is illustrated through both model-based simulations and experiments.

T. Chen and Y.-C. Wang present a fuzzy slack-diversifying fluctuation-smoothing rule to enhance the performance of a multiobjective job dispatching wafer fabrication factory. The performance of the proposed methodology which aims at optimizing cycle time, cycle time standard deviation, the maximum lateness, and number of tardy jobs is demonstrated through a model simulating a real wafer fabrication factory located in Taichung Scientific Park of Taiwan having a monthly capacity of about 25,000 wafers.

S. Cang et al. introduce an adaptive control system that can be employed to achieve projective synchronization of coupled chaotic systems which are not identical, with fully unknown parameters, uncertain terms, and external bounded disturbances. The design is based on the Lyapunov stability theory and the effectiveness of the proposed scheme is illustrated through two examples, namely, the Lorenz and Lü systems.

L. Tan and D. Lei provide analytic solutions for the steady state distribution of the vertex degree, the vertex strength, and the edge weight for a class of generalized weighted scalefree networks, where the new vertex connects to $m$ pairs of vertices selected preferentially. Numerical simulations are also provided and compared to the analytical derivations.

Y. Yao et al. formulate a delayed Internet worm propagation model with birth and death rates. It is shown through analysis that when the time delay passes a threshold, corresponding to a Hopf bifurcation, the worm propagation becomes unstable and gets out of control. The delay should thus be kept to values less than the threshold to ensure the stability of the system and a better predictability of the scale and rate of the worm spreading. Numerical experiments are presented, which fully support their analysis.

T. Wang et al. employ the immersed boundary-fictitious domain method to simulate numerically the adhesion and dissociation kinetics of erythrocytes in horizontal microchannels with stenosis. Their simulations demonstrate the importance of the geometrical characteristics on the dissociation of the erythrocyte aggregates and that the latter dissociate easier when they are horizontally and not vertically oriented.

Y. Zhao et al. investigate the cluster synchronization of impulsive complex networks with stochastic perturbation and time-varying delays. The nodes in the complex networks are nonidentical. Using the Lyapunov stability theory and stochastic analysis theory as well as linear matrix inequalities, sufficient conditions are derived to guarantee the cluster synchronization. Numerical simulations are provided to show the effectiveness of the theoretical results.

L. Wang et al. investigate the exponential synchronization problem for a class of moving agent networks in a twodimensional space, which exhibits time-varying topology structure. Based on the Lyapunov stability theory, adaptive feedback controllers are developed to guarantee the exponential synchronization between agents. A numerical example based on the Rössler system is provided to demonstrate the effectiveness and applicability of the proposed design approach.

B. Liu et al. studied the adaptive synchronization of complex dynamical networks satisfying local Lipschitz condition with switching topology. Based on differential inclusion and nonsmooth analysis, it is proved that all nodes can converge to a synchronous state even though only one node is informed, when introducing decentralized adaptive strategies to the coupling strengths and feedback gains. Numerical simulations are performed to illustrate the analytical results.

Constantinos I. Siettos Yannick De Decker Georgios C. Georgiou 


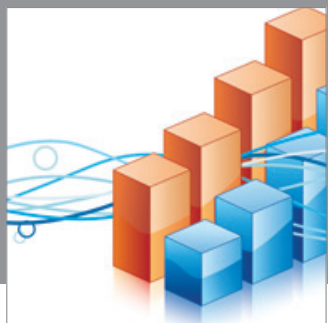

Advances in

Operations Research

mansans

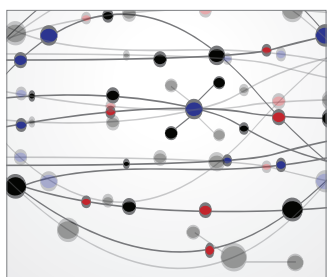

The Scientific World Journal
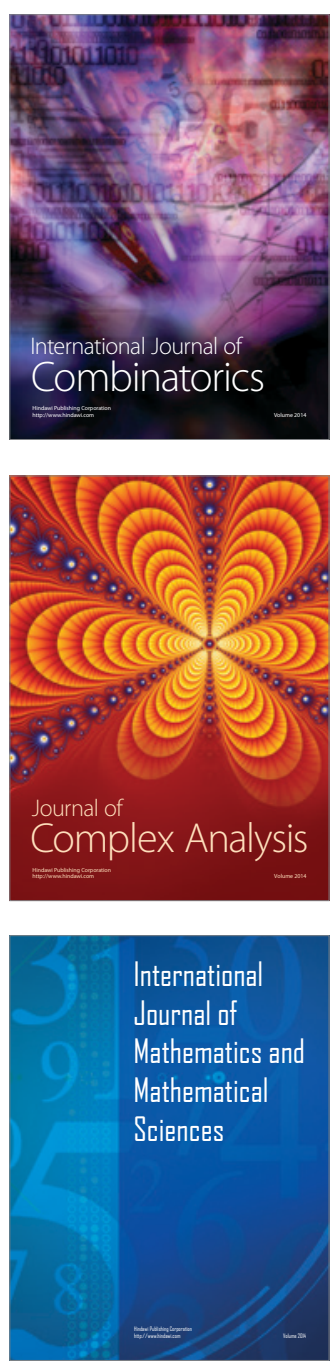
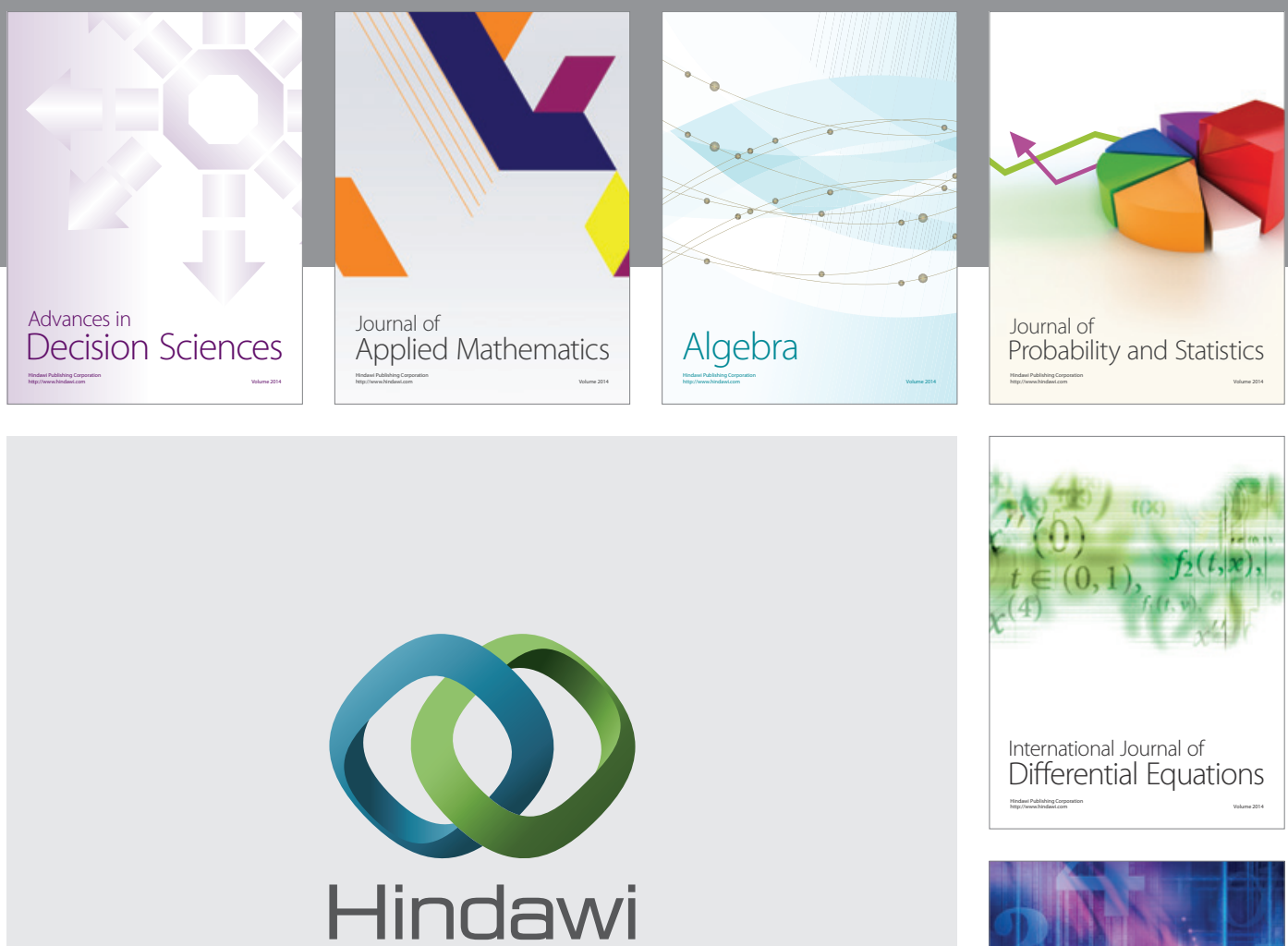

Submit your manuscripts at http://www.hindawi.com
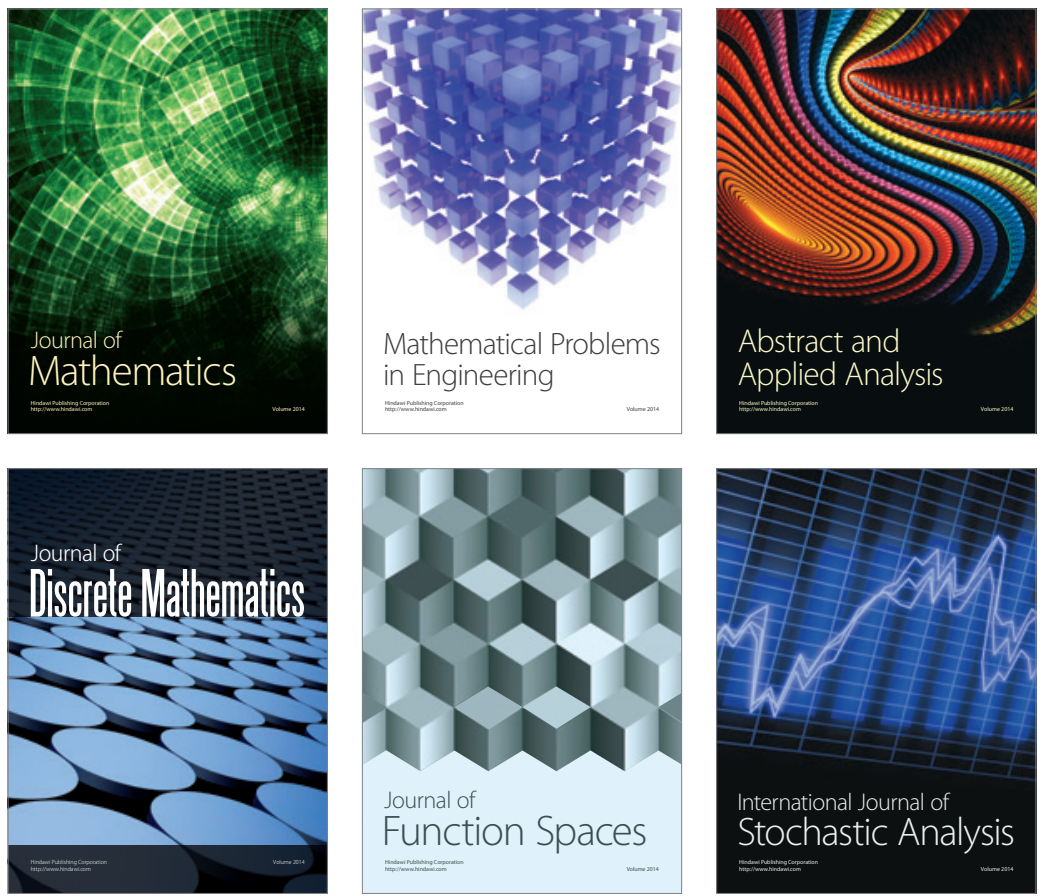

Journal of

Function Spaces

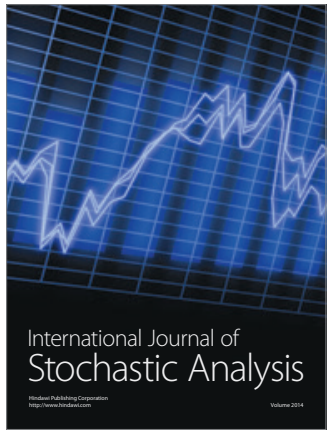

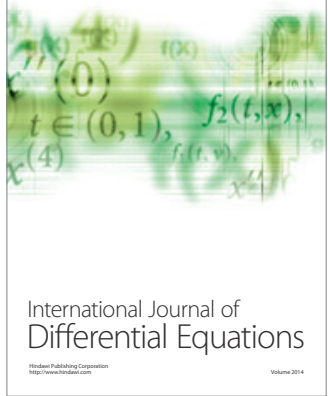
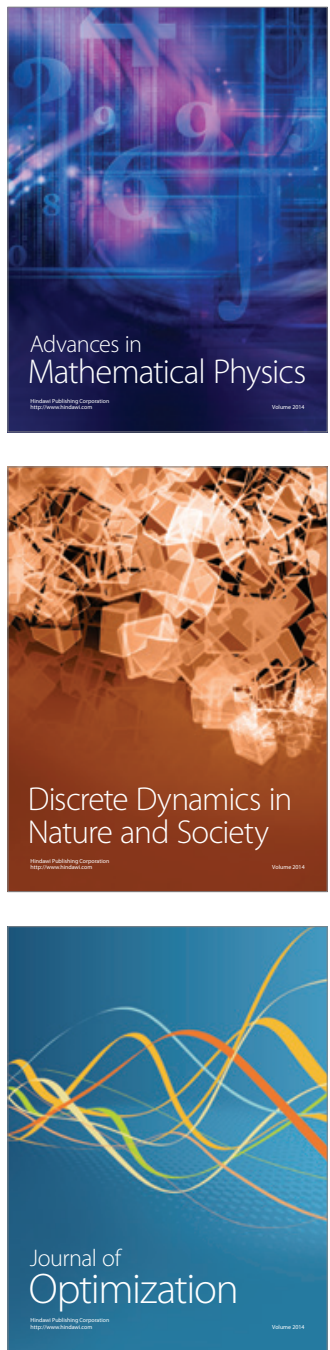